\title{
An Unusual Leptospira, Serotype illini (a New Serotype)
}

\author{
L. E. HANSON, D. N. TRIPATHY, L. B. EVANS, and A. D. ALEXANDER \\ College of Veterinary Medicine, University of Illinois, Urbana, Illinois 61801, and WHO/FAO \\ Leptospirosis Reference Laboratory, Division of Veterinary Medicine, Walter Reed Army Institute \\ of Research, Washington, D.C. 20012
}

\begin{abstract}
During the course of a periodic epidemiological survey for leptospirosis of a herd of bulls at the University of Illinois Agricultural Research Center, Dixon Springs, a new leptospiral strain was isolated from the urine of a clinically healthy Hereford bull, no. 3055. The strain was found to be antigenically unrelated to known pathogenic (Leptospira interrogans) and "saprophytic" (Leptospira bilflexa) serotypes. Phenotypically, it most closely resembled $L$. biflexa strains; however, in view of the reported distinct genetic characteristics of strain 3055, its species classification is not recommended at this time.
\end{abstract}

A periodic evaluation of leptospiral antibodies in a herd of 60 2-year-old Hereford bulls at the Dixon Springs Agricultural Center indicated recent Leptospira interrogans serotype pomona infection in the spring of 1965. (Information available since submission of the manuscript indicates "serovar" is the new official term for "serotype.") As no clinical signs of disease were evident, an attempt was made to isolate leptospiras from the urine of selected seropositive and seronegative bulls to determine whether serotype pomona was present in the herd. The isolation and characterization of a new leptospiral serotype from a clinically healthy bull (no. 3055) is described.

\section{MATERIALS AND METHODS}

Urine was collected from each animal in a sterile container, and approximately $0.1 \mathrm{ml}$ was transferred into $5 \mathrm{ml}$ of liquid and semisolid bovine albumin polysorbate 80 (4) medium. The inoculated medium was incubated at $30 \mathrm{C}$ and examined weekly over a 6-week period with dark-field microscopy for the presence of leptospires.

The leptospiral culture and the serum from the bull from which it was isolated were sent to the WHO/FAO Leptospirosis Reference Laboratory at Walter Reed Army Institue of Research (WRAIR) for typing and additional serological tests, respectively. The submitted culture was cloned by the use of plating medium. The cloned culture used as antigen was tested for cross-agglutination reactions with 85 different serotype-specific antisera selected from all known pathogenic serogroups, and with all except one (serotype tororo) of the antisera to the 55 classified Leptospira biflexa serotypes (2). Additional tests were made with antisera prepared against strain 3055 and against eight other unidentified "biflexa-like" isolates, four of which were from the same geographic region as strain 3055 . One of the latter strains, A-177, isolated from a turtle (R. D. Andrews, Ph.D. thesis, Univ. of Illinois, 1966), had the same growth, physiological, and phage characteristics as strain 3055 (H. E. Ellinghausen and A. Ritchie, personal communication), which it also resembled in genetic characteristics (unpublished data, WRAIR). The microscopic agglutination technique was used for serological tests (1). Homologous titers of test antisera ranged from $1: 10,000$ to $1: 500,000$.

Several differential tests, e.g., growth in 2,6-diaminopurine (8), growth at $13 \mathrm{C} \mathrm{(7),} \mathrm{hemolysis} \mathrm{of} \mathrm{mouse}$ erythrocytes $(9)$, and egg yolk reaction test (5), were conducted to determine the properties of strain 3055 .

\section{RESULTS}

A leptospiral strain was isolated in the bovine albumin polysorbate 80 medium from the urine of an asymptomatic bull (no. 3055) in March 1965. A subsequent attempt to isolate leptospires from the urine of bull 3055 was unsuccessful. In preliminary studies, strain 3055 was found to be antigenically unrelated to L. interrogans serotypes autumnalis, ballum, canicola, grippotyphosa, hyos, icterohaemorrhagiae, pomona, sejroe, georgia, and hardjo and to a few selected $L$. biflexa serotypes when tested against reference antisera. Serum from the bull had a homologous titer of 1:100 but did not react with serotypes autumnalis, grippotyphosa, canicola, icterohaemorrhagiae, pomona, or hardjo.

The initial serological findings were affirmed at WRAIR. Strain 3055 could not be serologically related to any of the known pathogenic and biflexa serotypes in extensive cross-agglutination testing. It reacted to titer $(1: 25,600)$ with the homologous antiserum and with 
antisera against A-177 and A-177 with anti3055 serum. Otherwise, only minor cross-reactions were elicited against antisera as follows: copenhageni, 1:200; mankarso, 1:400; ndahambukuje, 1:400; naam, 1:800; weaveri, $1: 400$; bindjei, 1:100; sofia, 1:100; worsfoldi, $1: 100$; mini, $1: 100$; djatzi, 1:100; bratislava, $1: 100 ;$ grippotyphosa, $1: 100$; canalzonae, $1: 100 ;$ garcia, $1: 500$; and fons, 1:500.

In differential tests the isolate grew well in the presence of $10 \mu \mathrm{g}$ of 2,6-diaminopurine per $\mathrm{ml}$ and at $13 \mathrm{C}$. Old cultures of this isolate hemolyzed mouse as well as rabbit, horse, hamster, and gerbil erythrocytes to varying titers. Lower titers were obtained with horse and rabbit erythrocytes $(1: 4$ to $1: 16)$ than with mouse, hamster, or gerbil erythrocytes (1:32 to $1: 64)$. It decomposed egg yolk medium in 3 to 4 days.

\section{DISCUSSION}

Strain 3055 not only is serologically distinct but also has remarkable genetic and cultural properties. Brendle et al. (3) found that strain 3055 had no deoxyribonucleic acid (DNA) affinities with known pathogenic and "saprophytic" genetic types of leptospires on the basis of DNA annealing tests and appeared to represent a new genetic "complex" of leptospires. Moreover, its guanine and cytosine content, $53 \mathrm{~mol} \%$, was found to be considerably higher than that of other leptospires (35.2 to $41.2 \mathrm{~mol} \%$ range) (3). Strain 3055 also represents the only leptospiral serotype in which a phagelike entity has been demonstrated (10). In addition, Ellinghausen (personal communication) reported that it differed from other leptospiras except strain A-177 in its ability to grow in ordinary Trypticase media. Although strain 3055 was isolated from a bull, it behaved like a non-pathogenic leptospira in pathogenicity tests (11) in its ability to grow at $13 \mathrm{C}$, in its relative resistance to 2,6-diaminopurine, 8-azaguanine, and $\mathrm{CuSO}_{4}$ (unpublished data, WRAIR), and in its hemolytic properties.

The close antigenic affinities of strain 3055 to strain A-177 was not surprising. Strain A-177 has the same cultural and physiological characteristics as those of strain 3055. Moreover, a phagelike entity has also been demonstrated in strain A-177 (Ritchie and Ellinghausen, personal communication). Like strain 3055 , it has a high guanine and cytosine base ratio (51.2 mol\%) (J. J. Brendle, M. Rogul, A. D. Alexander, unpublished data).

On the basis of serological findings, strain
3055 can be classified as a new serotype, for which we selected the name illini. The species classification of 3055 is problematical because it does not fit into either of the two species, $L$. interrogans ("pathogenic") and L. biflexa ("non-pathogenic"), recently proposed by the Leptospira Subcommittee, International Committee on Systematic Bacteriology (6). The two species were established on the basis of pathogenicity and other phenotypic differences as well as genetic differences as determined by DNA-DNA annealing tests. Although strain 3055 is phenotypically similar to L. biflexa strains, it cannot be classified in these species in view of its remarkably distinct genetic characteristics. Accordingly, at this time we do not propose species classification of strain 3055 .

\section{ACKNOWLEDGMENTS}

We are grateful to Marina Cinco, Istituto di Microbiologia, Universita di Trieste, Trieste, Italy, for making available various $L$. biflexa serotype antisera, and for conducting serological tests for serotype illini antibodies on select $L$. biflexa serotypes.

Technical assistance of R. Marlowe and W. Manuel is gratefully acknowledged.

This investigation was supported in part by funds from Federal Hatch grant 70-302 and grant CC 00190 from the Center for Disease Control.

\section{LITERATURE CITED}

1. Alexander, A. D. 1970. Leptospira, p. 224-250. In J. E, Blair, E. H. Lennette, and $J_{n}$ P. Truant (ed.), Manual of clinical microbiology. American Society for Microbiology, Bethesda, Md.

2. Babudiere, B. 1972. List of leptospira strains kept in the WHO/FAO Leptospira Reference Laboratory in Rome. Ann. Inst. Super. Sanita 8:159196.

3. Brendle, J. J., M. Rogul, and A. D. Alexander. 1974. Deoxyribonucleic acid hybridization among selected leptospiral serotypes. Int. J. Syst. Bacteriol. 24:205-214.

4. Ellinghausen, H. C., Jr., and W. G. McCullough. 1965. Nutrition of Leptospira pomona and growth of 13 other serotypes: fractionation of oleic albumin complex and a medium of bovine albumin and polysorbate 80 . Amer. J. Vet. Res. 26:45-51.

5. Füzi, M., and R. Ssoka. 1961. An egg-yolk reaction test for the differentiation of leptospirae. J. Pathol. Bacteriol. 82:208-212.

6. International Committee on Systematic Bacteriology. Subcommittee on the Taxonomy of Leptospira. 1974. Minutes of the meeting, 30 August -4 September 1973. Int. J. Syst. Bacteriol. 24:381382.

7. Johnson, R. C., and V. G. Harris. 1967. Differen- 
tiation of pathogenic and saprophytic leptospires. I. Growth at low temperatures. J. Bacteriol. 94:27-31.

8. Johnson, R. C., and V. G. Harris. 1968. Purine analogue sensitivity and lipase activity of leptospires. Appl. Microbiol. 16:1584-1590.

9. Pentek-Juhasz, M. 1960. Untersuchungen über Die Hemolytische Eigenschaft von Leptospira biflexa-stammen. Acta Vet. Acad. Sci. Hung.
10:233-237.

10. Ritchie, A. E., and H. C. Ellinghausen. 1969. Bacteriophage-like entities associated with a leptospire, p. 228-229. In J. Arcenaux (ed.), Proceedings of the Electron-Microscopy Society of America. Claitor's Publishing Div., Baton Rouge.

11. Tripathy, D. N., and L. E. Hanson. 1973. Studies of Leptospira illini strain 3055: pathogenicity for different animals. Amer. J. Vet. Res. 34:557-562. 